\title{
Iatrogenic hypomagnesemia: an underestimated clinical problem
}

\author{
Antonio Villa, ${ }^{1}$ Paolo Zanada, ${ }^{2}$ Adriana Pellegrino, ${ }^{1}$ Gabriella Nucera, ${ }^{1}$ Elena Martinoli, ${ }^{1}$ Annamaria Boschiero, ${ }^{1}$ \\ Francesca Campanini ${ }^{1}$
}

${ }^{1}$ Department of Emergency Medicine; and ${ }^{2}$ Laboratory of Analyses, Fatebenefratelli Hospital, Milano, Italy

\begin{abstract}
Hypomagnesemia is defined by having a serum magnesium level of less than $1.7 \mathrm{mg} / \mathrm{dL}$. The magnesium balance is tightly regulated by the concerted actions of the intestine, bone and kidneys. This balance can be disturbed by a wide variety of drugs. Recently, we observed a case of severe hypomagnesemia caused by proton pump inhibitor therapy. We conducted a retrospective study of an intrahospital population to evaluate the prevalence of hypomagnesemia and the relationship with associated drugs. Among 181 patients with hypomagnesemia only 29 were found to have hypomagnesemia with specific causes, such as chronic diarrhea, vomiting, and so on. In the remaining patients, 120 have taken proton pump inhibitors and/or diuretics and/or metformin. Clinicians should consider proton pump inhibitors as a possible causative agent when investigating hypomagnesemia and they should be especially attentive with patients who take proton pump inhibitors, especially in cases of long-term therapy ( $\geq 1$ year) and/or concomitant administration of other agents that may lower magnesium levels (e.g., diuretics or metformin).
\end{abstract}

\section{Introduction}

Hypomagnesemia is a common disturbance occurring in up to $12 \%$ of hospitalised patients. ${ }^{1}$ Hypomagnesemia may result from an inadequate intake of magnesium, intracellular shift (treatment of diabetic ketoacidosis, refeeding syndrome, and hungry bone syndrome), increased gastrointestinal loss (chronic diarrhea, malabsorption syndrome, steatorrhea, vomiting, and nasogastric suction), or most common, increased losses of urine.

In addition, a number of drugs are known to cause hypomagnesemia. Drugs frequently associated with renal magnesium wasting include loop and thiazide diuretics, aminoglycosides, amphotericin, cisplatin, cyclosporine, pentamidine and foscarnet. ${ }^{2}$ Also met-

Correspondence: Antonio Villa, via Fiuggi 56, 20159 Milano, Italy.

Mobile: +39.338 .6562521 .

E-mail: antonio_villa@fastwebnet.it

Key words: Hypomagnesemia; proton pump inhibitors; metformin; diuretics.

Received for publication: 11 June 2014.

Revision received: 8 August 2014.

Accepted for publication: 28 August 2014.

This work is licensed under a Creative Commons Attribution NonCommercial 3.0 License (CC BY-NC 3.0).

(C) Copyright A. Villa et al., 2015

Licensee PAGEPress, Italy

Italian Journal of Medicine 2015; 9:287-289

doi:10.4081/itjm.2015.521 formin can reduce serum magnesium, ${ }^{3}$ with a complex mechanism; moreover, an increasing number of reports have recently identified proton pump inhibitors (PPIs) as a cause of hypomagnesemia..$^{4-9}$

We recently observed an analogous case in a 78year old man with peripheral neurological symptoms and serum magnesium level of $0.84 \mathrm{mg} / \mathrm{dL}$. When pantoprazole, which he had been taking over a period, was stopped he recovered and magnesium levels normalized (personal communication, 2014).

We carried out a retrospective analysis of an intrahospital population to assess the frequency of hypomagnesemia and the relationship with associated drugs.

\section{Materials and Methods}

From the laboratory database of 2013 we have extracted data related to serum magnesium analysis. In the patients with hypomagnesemia $(<1.70 \mathrm{mg} / \mathrm{dL})$ we analyzed clinical documentation with special attention to symptoms and diseases that could influence magnesium metabolism (diabetes, renal diseases, gastrointestinal disorders, alcohol assumption) and drugs that lower serum magnesium.

\section{Results}

In 2013, 1555 serum magnesium evaluations were conducted in 1064 in-hospital patients.

In 181 patients hypomagnesemia was diagnosed $(17 \%)$. The concomitant pathologies in these patients were: diabetes 42, alcoholic assumption 2, Crohn's disease 2 , hepatic cirrhosis 1 , renal disease 10 , gastrointestinal disorders (vomiting and/or diarrhea) 14. 
In two patients cardiovascular arrest occurred, in one patient was registered asystole, in one atrial tachycardia, in two palpitations without electrocardiogram changes were reported and two patients had seizures.

Among 181 patients with hypomagnesemia, only 29 were noted to have hypomagnesemia in the presence of a clinical cause. In the remaining 152 patients without specific causes of hypomagnesemia, 110 (72\%) had been taking PPI and/or diuretics and/or metformin. Fifty-seven only PPI, 9 only diuretics and one only metformin.

\section{Discussion and Conclusions}

Magnesium, the second most common intracellular cation, is of central importance for a broad range of physiological processes, including intracellular signaling, neuronal excitability, muscle contraction, bone formation and enzyme activation..$^{10}$ Clinical manifestations of hypomagnesemia involve neuromuscular hyperexcitability that may range from tremors, fasciculation, tetany, ataxia, to seizures, and neuropsychiatric disturbances including apathy, delirium, and even coma. ${ }^{11}$ Other potentially life-threatening complications include arrhythmias (atrial fibrillation, ventricular tachycardia, ventricular fibrillation, and torsades de pointes), enhanced sensitivity to digoxin toxicity, and sudden death. ${ }^{12,13}$

Hypomagnesemia is relatively common in hospitalised patients, particularly in intensive care units, ${ }^{12}$ to our knowledge this is the first report about the prevalence of hypomagnesemia in a large hospitalized Italian population.

Drugs can interfere with magnesium homeostasis in multiple ways.

Our data show that many patients can have hypomagnesemia in the absence of a clinical cause. A retrospective analysis of clinical documentation shows that an important number of these patients have taking some drugs that are known to influence magnesium homeostasis.

Diuretics may cause hypermagnesuria, leading to possible hypomagnesemia and magnesium depletion. ${ }^{10}$

Loop diuretics act in the thick ascending limb of the nephron, where $40-70 \%$ of the filtered magnesium is reabsorbed; the use of loop diuretics may lead to renal magnesium wasting and hypomagnesemia due to reduced paracellular magnesium reabsorption. ${ }^{10}$

The effect of thiazides on magnesium reabsorption becomes apparent only upon chronic treatment. Hypomagnesemia has been suggested to result from potassium deficiency, increased passive magnesium secretion or decreased active magnesium reabsorption in the distal convolute tubule. ${ }^{10}$

Low serum magnesium concentrations can be found in patients with diabetes. ${ }^{14}$ This association may reflect a vicious cycle with hyperinsulinemia associated with insulin resistance contributing to extracellular magnesium depletion and, in turn, further augmentation of insulin resistance by hypomagnesemia. ${ }^{15}$ Metformin reduces serum magnesium ${ }^{3,16}$ and maintains diabetes-associated hypomagnesemia. ${ }^{17}$ The influence of metformin on magnesium homeostasis is, however, complex, with evidence of an increase in intracellular magnesium. ${ }^{17,18}$

PPIs belong to the most widely prescribed classes of drugs in the world. In 2006, two cases of PPI-induced hypomagnesemia were published by Epstein and coll. ${ }^{4}$ Since then, several groups have described similar findings in patients using PPIs. ${ }^{5,6,8,12,19}$ The exact prevalence remain unknown; however, the growing number of case reports indicated that these patients might represent the tip of an iceberg. ${ }^{10}$

Long-term use seems to play a role, as all patients were using PPIs for more than 1 year. Remarkably, patients recover relatively quickly upon discontinuation and relapse within days when medication is restarted. This could indicate that PPIs might have a relatively subtle effect on magnesium homeostasis, which only becomes overt when magnesium stores are completely depleted. ${ }^{10}$ Urinary magnesium excretion is low, indicating intact renal function. Taken together, these findings point towards an intestinal abnormality as the most likely problem underlying PPI-induced hypomagnesemia.

Several explanations have been suggested in the published case reports. First, magnesium absorption might depend on the stomach's acidity for solubilisation. Achlorhydria caused by PPIs could, therefore, reduce magnesium availability for absorption, resulting in diminished magnesium absorbed in the intestine. A second explanation would be an increase in intestinal magnesium loss via secretion; considerable amounts of magnesium can be excreted in the intestine, and thus a shift in the balance between magnesium absorption and secretion in the intestine could result in net magnesium loss..$^{10}$ Thirdly, active transcellular or passive paracellular magnesium absorption could be impaired. The transient receptor potential melastin (TRPM) 6 and 7 cation channels are thought to be responsible for magnesium transport into the cell. TRPM6 activity is positively regulated by extracellular protons, and an inhibitory effect of PPIs on the colonic $\mathrm{H}^{+} / \mathrm{K}^{+}$-ATPase (a homologue of the gastric $\mathrm{H}^{+} / \mathrm{K}^{+}$-ATPase that is inhibited by PPIs) could reduce TRPM6 activity due to a decreased level of excreted protons. ${ }^{10}$

Physicians should be aware of this serious side effect of PPIs and should obtain blood magnesium levels prior to initiation of PPIs when patients are expected to be on treatment for a long period of time and in those with other potential causes of hypomagnesemia. ${ }^{20}$

Patients suffering from hypomagnesemia due PPIs 
can be treated with supplements or switched to a histamine $\mathrm{H}_{2}$ receptor antagonist.

Clinician should consider PPIs as a possible causative agent when investigating hypomagnesemia. In order to take immediate and effective action they should suspect hypomagnesemia in patients who take PPIs, especially in cases of long-term therapy $(\geq 1$ year) and/or concomitant administration of other agents that may lower magnesium levels (e.g., diuretics or metformin).

\section{References}

1. Wong ET, Rude RK, Singer FR, Shaw ST Jr. A high prevalence of hypomagnesemia and hypermagnesemia in hospitalized patients. Am J Clin Pathol 1983;79: 348-52.

2. Florentin M, Elisaf MS. Proton pump inhibitor-induced hypomagnesemia: a new challenge. WJN 2012;1:151-4.

3. Walti MK, Zimmermann MB, Spinas GA, Hurrel RF. Low plasma magnesium in type 2 diabetes. Swiss Med Wkly 2003;133:289-92.

4. Epstein M, McGrath S, Law F. Proton-pump inhibitors and hypomagnesemic hypo-parathyroidism. N Engl J Med 2006;355;1834-6.

5. Cundy T, Mackay J. Proton pump inhibitors and severe hypomagnesemia. Curr Opin Gastroenterol 2011;27:180-5.

6. Kuipers MT, Thang HD, Arntzenius AB. Hypomagnesemia due to use of proton pump inhibitors - a review. Neth J Med 2009;67:169-72.

7. Mackay JD, Bladon PT. Hypomagnesemia due to proton-pump inhibitor therapy: a clical case series. QJM 2010;103:387-95.

8. Hoorn EJ, van der Hock J, de Man RA, et al. A case series of proton pump inhibitor-induced hypomagnesemia. Am J Kidney Dis 2010;56:112-6.

9. Cundy T, Dissanayake A. Severe hypomagnesemia in long-term users of proton-pump inhibitors. Clin Endocrinol 2008;69:338-41.

10. Lameris AL, Monnens LA, Bindels RJ, Hoenderop JGJ. Drug-induced alterations in $\mathrm{Mg}^{2+}$ homeostasis. Clin Sci 2012;123:1-14.

11. Pham PC, Pham PA, Pham SV, et al. Hypomagnesemia: a clinical perspective. Int J Nephrol Renovasc Dis 2014; 7:219-30.

12. Regolisti G, Cabassi A, Parenti E, et al. Severe hypomagnesemia during long-term treatment with a proton pump inhibitors. Am J Kidney Dis 2010;56:168-74.

13. El-Charabaty E, Saifan C, Abdallah M, et al. Effects of proton pump inhibitors and eletrolyte disturbances on arrhythmias. Int J Gen Med 2013;6:515-8.

14. Pham PC, Pham PM, Pham SV, et al. Hypomagnesemia in patients with type 2 diabetes. Cli J Am Soc Nephrol 2007;2:366-73.

15. Chaudhary DP, Sharma R, Bansal DD. Implications of magnesium deficiency in type 2 diabetes: a review. Biol Trace Elem Res 2010;134:119-29.

16. Ma J, Folsom AR, Melnick SL, et al. Associations of serum and dietary magnesium with cardiovascular disease, hypertension, diabetes, insulin and carotid arterial wall thickness: the RIC study. Atherosclerosis Risk in Communities Study. J Clin Epidemiol 1995;48:927-40.

17. Dosa MD, Hangan LT, Crauciuc E, et al. Influence of therapy with metformin on the concentration of certain divalent actions in patients with non-insulin-dipendent diabetes mellitus. Biol Trace Elem Res 2011;142:36-46.

18. Gorelik O, Efrati S, Berman S, et al. Effect of various clinical variables on total intracellular magnesium in hospitalized normomagnesemic diabetic patients before discharge. Biol Trace Elem Res 2007;120:102-9.

19. Furlanetto TW, Faulhaber GA. Hypomagnesemia and proton pump inhibitors: below the tip of the iceberg. Arch Intern Med 2011;171:1391-2.

20. Famularo G, Gasbarrone L, Minisola G. Hypomagnesemia and proton-pump inhibitors. Expert Opin Drug Saf 2013;12:709-16. 